ANALELE ŞTIINŢIFICE ALE UNIVERSITĂŢII “AL.I. CUZA” DIN IAŞI (S.N.)

MATEMATICA, Tomul LXI, 2015, f.1

DOI: $10.2478 /$ aicu-2014-0027

\title{
SOME CURVATURE IDENTITIES ON GRADIENT SHRINKING CONFORMAL RICCI SOLITON
}

\author{
BY
}

\begin{abstract}
ARINDAM BHATTACHARYYA and NIRABHRA BASU*
\end{abstract}
\begin{abstract}
In this paper we have established some curvature identities for gradient shrinking conformal Ricci soliton.

Mathematics Subject Classification 2010: 53C44, 35K65, 58D17.

Key words: Ricci flow, Ricci soliton, curvature operator, conformal Ricci flow.
\end{abstract}

\section{Introduction}

HAMILTON introduced the study of the Ricci flow [4] in the year 1982. After that Ricci flow has become a powerful tool for the study of Riemannian manifolds, especially for those manifolds with positive curvature. Perelman did excellent work on Ricci flow [6], [7]. He used Ricci flow and its surgery to prove Poincaré conjecture. In the general case, the solution of the Ricci flow may have much more complicated behavior and develop singularities in finite time, in particular the curvature may become arbitrarily large in some region while staying bounded in its complement. For example, if one starts with an almost round cylindrical neck, which looks like $S^{2} \times E^{1}$ connecting two large pieces of low curvature, then the positive curvature in the $S^{2}$ direction will dominate the slightly negative curvature in the $E^{1}$ direction and therefore one expects the neck to shrink and pinch off. Singularities can be removed by surgeries.

FisCHER developed the concept of conformal Ricci flow [3] during 20032004. In classical Ricci flow equation the unit volume constraint plays

\footnotetext{
${ }^{*}$ Supported by the grant of CSIR Sanction No 09/096(0656)/2010-EMR-I.
} 
an important role but in conformal Ricci flow equation scalar curvature is considered. This Ricci flow equations are called conformal Ricci flow because of the role that conformal geometry plays in constraining the scalar curvature. Also these equations are the vector field sum of a conformal flow equation and a Ricci flow equation. Let $M$ be a smooth closed connected oriented $n$-manifold $(n>3)$. The conformal Ricci flow equation on $M$ are defined by the equation

$$
\frac{\partial g_{i j}}{\partial t}=-2 R_{i j}-g_{i j}\left(\frac{2}{n}+p\right), R(g)=-1,
$$

where $p$ is scalar non dynamical field (time dependent scalar field).

A Ricci soliton emerges as the limit of the solutions of the Ricci flow. A solution to the Ricci flow is called Ricci soliton if it moves only by a one parameter group of diffeomorphism and scaling. For example, we can consider Hamilton's ciger soliton [2], [5]. Hamilton's cigar soliton is the complete Riemannian surface $\left(E^{2}, g\right)$; where $g=\frac{d x^{2}+d y^{2}}{1+x^{2}+y^{2}} ; d x^{2}=d x \otimes d x$. It is called a cigar because it is asymptotic to a cylinder at infinity, has maximal Gauss curvature at the origin and burns away.

A complete Riemannian manifold $\left(M^{n}, g_{i j}\right)$ is a gradient Ricci soliton if there exists a smooth function $f$ on $M$ such that $R_{i j}+\nabla_{i} \nabla_{j} f=\rho g_{i j}$ for some constant $\rho$. $f$ is called a potential function of the Ricci soliton. When $\rho=0$, it is called steady soliton; when $\rho>0$ the soliton is called shrinking soliton and for $\rho<0$ it is called expanding soliton. If $f$ is constant then the above equation becomes $R_{i j}=\rho g_{i j}$, i.e. the manifold becomes Einstein. So for that case the solutions are uniformly shrinking or expanding depending upon $\rho$. We consider Ricci solitons for more general notions of self similar solutions without taking the manifold as compact. Ricci soliton exhibit rich geometric properties.

In particular a gradient shrinking Ricci soliton satisfies the equation $R_{i j}+\nabla_{i} \nabla_{j} f-\frac{1}{2 \tau} g_{i j}=0$, where $\tau=T-t$ and $T$ is the time when the soliton deforms into a point, $f$ is the Ricci potential function.

For conformal Ricci flow, if the vector field which introduced the diffeomorphism is in fact the gradient of a function $f$ then we call it a gradient shrinking conformal Ricci soliton. For conformal gradient Ricci soliton the equation will be

$$
R_{i j}+\nabla_{i} \nabla_{j} f=\left(\frac{1}{2 \tau}-\frac{2}{n}-p\right) g_{i j}
$$


For a conformal gradient shrinking Ricci soliton, $\left(\frac{1}{2 \tau}-\frac{2}{n}-p\right)>0$, which implies $n<\frac{4 \tau}{1-2 \tau p}$. Also we know that $n>3$, so we have $3<n<\frac{4 \tau}{1-2 \tau p}$. By inner multiplication to (1.2) by $g^{i j}$ we obtain

$$
R+\Delta f=\frac{n}{2 \tau}-2-p n
$$

For conformal Ricci flow $R=-1$, so we have

$$
\Delta f=\frac{n}{2 \tau}-1-p n .
$$

From equation (4.13) of [1] and [2] we have for a gradient shrinking Ricci soliton $R+|\nabla f|^{2}=\frac{f-c}{\tau}$, where $c$ is a constant in space. Now for conformal Ricci flow when $R=-1$ we have

$$
|\nabla f|^{2}=\frac{f-c}{\tau}+1
$$

Thus we can state the following result.

Result 1.1. For a conformal gradient shrinking Ricci soliton we have $\Delta f=\frac{n}{2 \tau}-1-p n$ and $|\nabla f|^{2}=\frac{f-c}{\tau}+1$. From [1] we have $(\operatorname{div} R m)_{j k l}=$ $R_{i j k l, i}=\nabla_{i} R_{i j k l}$. Using Bianchi identity, $\nabla_{i} R_{k l j i}=-\nabla_{k} R_{i j l i}-\nabla_{l} R_{i j i k}$. We know $R_{i j l i}=g_{i m} R_{j l i}^{m}$. Therefore

$$
\nabla_{i} R_{k l j i}=-\nabla_{k} R_{j l}-\nabla_{l} R_{j k} .
$$

Using (1.2) we get $\nabla_{k} R_{j l}+\nabla_{k} f_{j l}=0$. Where $f_{k}=\nabla_{k} f$, which implies

$$
\nabla_{k} R_{j l}=-\nabla_{k} f_{j l} .
$$

Similarly

$$
\nabla_{l} R_{j k}=-\nabla_{l} f_{j k}
$$

From (1.7) and (1.8) we can write

$$
-\nabla_{k} f_{j l}+\nabla_{l} f_{j k}=\nabla_{l} \nabla_{k} f_{j}-\nabla_{k} \nabla_{l} f_{j}=R_{l k j p} f_{p} .
$$

Hence as obtained in [1]

$$
\nabla_{i}\left(R_{i j k l} e^{-f}\right)=0
$$


and

$$
\nabla_{i}\left(R_{i j} e^{-f}\right)=0 .
$$

And also using integration by parts, we derive that

$$
\int|\operatorname{div} R m|^{2} e^{-f}=\int\left(R_{j l, k}-R_{j k, l}\right)\left(R_{j l, k}-R_{j k, l}\right) e^{-f}
$$

We shall now prove some identities by using these results.

2. Identities on Riemannian curvature under gradient shrinking conformal Ricci soliton

In this section we derive some curvature identities on conformal gradient shrinking Ricci soliton.

Lemma 2.1. On a compact gradient shrinking conformal Ricci soliton

$$
\int R_{k l j p} R_{k j} f_{l p} e^{-f}=-\frac{1}{2} \int|\operatorname{div} R m|^{2} e^{-f}
$$

Proof. Proof follows as given in [1].

Theorem 2.1. On a compact gradient shrinking conformal Ricci soliton $\int R m(R c, R c) e^{-f}=\left(\frac{1}{2 \tau}-\frac{2}{n}-p\right) \int|R c|^{2} e^{-f}+\frac{1}{2} \int|\operatorname{div} R m|^{2} e^{-f}$.

Proof. From (1.2) we have $f_{k p}=\left(\frac{1}{2 \tau}-\frac{2}{n}-p\right) g_{k p}-R_{k p}$.

Now using the above Lemma and (1.2) we get

$$
\begin{aligned}
\int|\operatorname{div} R m|^{2} e^{-f} & =-2 \int R_{l k j p} R_{l j}\left[\left(\frac{1}{2 \tau}-\frac{2}{n}-p\right) g_{k p}-R_{k p}\right] e^{-f} \\
& =-2\left(\frac{1}{2 \tau}-\frac{2}{n}-p\right) \int|R c|^{2} e^{-f}+2 \int R m(R c, R c) e^{-f}
\end{aligned}
$$

so, we have $\int R m(R c, R c) e^{-f}=\left(\frac{1}{2 \tau}-\frac{2}{n}-p\right) \int|R c|^{2} e^{-f}+\frac{1}{2} \int|\operatorname{div} R m|^{2} e^{-f}$.

\section{Lemma 2.2.}

$$
\nabla_{i} \nabla_{j} R_{i k}-\nabla_{j} \nabla_{i} R_{i k}=R_{j m} R_{m k}-R_{i j m k} R_{i m} .
$$

Proof. We know Ricci identity in 2 - form is $\nabla_{i} \nabla_{j} R_{l k}-\nabla_{j} \nabla_{i} R_{l k}=$ $-R_{i j l}^{m} R_{m k}-R_{i j k}^{m} R_{l m}$. Lowering the indices in the right hand side and 
putting $i=l$ in both the sides and taking the sum we get our desired result as obtained in [1].

Lemma 2.3. On a compact gradient shrinking conformal Ricci soliton

$$
\int \nabla_{k} R_{j l} \nabla_{l} R_{j k} e^{-f}=\int R m(R c, R c) e^{-f}-\left(\frac{1}{2 \tau}-\frac{2}{n}-p\right) \int|R c|^{2} e^{-f}
$$

Proof. From (2.1) of [1] we have

$$
\begin{aligned}
-2 \int \nabla_{k} R_{j l} \nabla_{l} R_{j k} e^{-f} & =2 \int R_{j k}\left(\nabla_{i} \nabla_{j} R_{i k}-\nabla_{j} R_{i k} f_{i}\right) e^{-f} \\
& =2 \int R_{j k}\left(\nabla_{i} \nabla_{j} R_{i k}\right) e^{-f}-2 \int R_{j k}\left(\nabla_{j} R_{i k} f_{i}\right) e^{-f} .
\end{aligned}
$$

Now using Lemma 2.2, we have, as obtained in [1]

$$
\begin{aligned}
& -2 \int \nabla_{k} R_{j l} \nabla_{l} R_{j k} e^{-f}=2 \int R_{j k}\left(\nabla_{j} \nabla_{i} R_{i k}+R_{m j} R_{m k}-R_{i j m k} R_{i m}\right) e^{-f} \\
& +2 \int R_{i k} R_{j k} f_{i j} e^{-f}=2 \int R_{j k} \nabla_{j}\left(\nabla_{i} R_{i k}\right) e^{-f}+2 \int R_{j k} R_{m j} R_{m k} e^{-f} \\
& -2 \int R_{j k} R_{i j m k} R_{i m} e^{-f}+2 \int R_{i k} R_{j k} f_{i j} e^{-f} .
\end{aligned}
$$

Using (1.11) we have

$$
\begin{aligned}
-2 \int \nabla_{k} R_{j l} \nabla_{l} R_{j k} e^{-f} & =2 \int R_{j k} R_{m j} R_{m k} e^{-f} \\
& +2 \int R_{i k} R_{j k} f_{i j} e^{-f}-2 \int R_{j k} R_{i j m k} R_{i m} e^{-f} \\
& =2 \int R_{j k} R_{k i}\left(f_{i j}+R_{i j}\right) e^{-f}-2 \int R_{i j m k} R_{i m} R_{j k} e^{-f}
\end{aligned}
$$

From (1.2) and above equation we get $-2 \int \nabla_{k} R_{j l} \nabla_{l} R_{j k} e^{-f}=2 \int R_{j k} R_{k i}\left[\left(\frac{1}{2 \tau}-\right.\right.$ $\left.\left.\frac{2}{n}-p\right) g_{i j}-R_{i j}+R_{i j}\right]-2 \int R_{i j m k} R_{i m} R_{j k} e^{-f}=2\left(\frac{1}{2 \tau}-\frac{2}{n}-p\right) \int|R c|^{2} e^{-f}-$ $2 \int R m(R c, R c) e^{-f}$.

Thus we obtain the result $\int \nabla_{k} R_{j l} \nabla_{l} R_{j k} e^{-f}=\int R m(R c, R c) e^{-f}-\left(\frac{1}{2 \tau}-\right.$ $\left.\frac{2}{n}-p\right) \int|R c|^{2} e^{-f}$.

Lemma 2.4. On a compact gradient shrinking conformal Ricci soliton $\int \nabla_{j} \nabla_{i} R_{i k} R_{j k} e^{-f}=0$. 
Proof. From definition of covariant differentiation and (1.11) we have, as obtained in [1] $\int \nabla_{j}\left(\nabla_{i} R_{i k} R_{j k}\right) e^{-f}=-\int \nabla_{i} R_{i k} \nabla_{j}\left(R_{j k} e^{-f}\right)=0$. As a consequence of the above lemmas we can state the following theorem.

Theorem 2.2. On a compact gradient shrinking conformal Ricci soliton we have $\int R m(R c, R c) e^{-f}=\int|\nabla R c|^{2} e^{-f}+\left(\frac{1}{2 \tau}-\frac{2}{n}-p\right) \int|R c|^{2} e^{-f}-$ $\frac{1}{2} \int|\operatorname{div} R m|^{2} e^{-f}$.

Proof. $\int|\operatorname{div} R m|^{2} e^{-f}=\int\left|\nabla_{k} R_{j l}-\nabla_{l} R_{j k}\right|^{2} e^{-f}=2 \int|\nabla R c|^{2} e^{-f}-$ $2 \int \nabla_{k} R_{j k} \nabla_{l} R_{j k} e^{-f}$. By using Lemma 2.3, we obtain $-2 \int \nabla_{k} R_{j l} \nabla_{l} R_{j k} e^{-f}$ $=2\left(\frac{1}{2 \tau}-\frac{2}{n}-p\right) \int|R c|^{2} e^{-f}-2 \int R m(R c, R c) e^{-f}=2 \int|\nabla R c|^{2} e^{-f}+2\left(\frac{1}{2 \tau}-\right.$ $\left.\frac{2}{n}-p\right) \int|R c|^{2} e^{-f}-\int|\operatorname{div} R m|^{2} e^{-f}$. Therefore

$$
\begin{aligned}
\int R m(R c, R c) e^{-f} & =\int|\nabla R c|^{2} e^{-f}+\left(\frac{1}{2 \tau}-\frac{2}{n}-p\right) \int|R c|^{2} e^{-f} \\
& -\frac{1}{2} \int|\operatorname{div} R m|^{2} e^{-f}
\end{aligned}
$$

\section{An identity on Ricci curvature under gradient shrinking conformal Ricci soliton}

In this section we prove an identity on Ricci curvature under conformal gradient shrinking Ricci soliton.

Theorem 3.1. On a compact gradient shrinking conformal Ricci soliton $\int g^{l i}|R c|^{2} \Delta e^{-f}=\int g^{l i}|\nabla R c|^{2} e^{-f}+\int K g^{l i} e^{-f}-\int g^{l i} \nabla_{i} R_{i l k j} f_{l} R_{j k} e^{-f}-\left(\frac{n}{2 \tau}-\right.$ $1-p n) \int R_{i l k j} R_{j k} e^{-f}$.

Proof. From (1.7) and (1.8) we get $\nabla_{j} R_{i k}=-\nabla_{j} f_{i k}$ and $\nabla_{i} R_{j k}=$ $-\nabla_{i} f_{j k}$.

Now from (1.9) we have $-\nabla_{j} f_{i k}+\nabla_{i} f_{j k}=R_{k j i l} f_{l}$. Combining those results we have

$$
\nabla_{i} R_{j k}=\nabla_{j} R_{i k}-R_{k j i l} f_{l}
$$

Now $\Delta R_{j k}=\nabla_{i}\left(\nabla_{i} R_{j k}\right)$ Using (3.1) we have

$$
\Delta R_{j k}=\nabla_{i} \nabla_{j} R_{j k}-\left(\nabla_{i} R_{k j i l}\right) f_{l}-R_{k j i l} f_{l i}
$$


so,

$<\Delta R c, R c>=\nabla_{i} \nabla_{i} R_{j k} R_{j k}=\nabla_{i}\left(\nabla_{i} R_{j k}\right) R_{j k}=\nabla_{i}\left(\nabla_{j} R_{i k}-R_{k j i l} f_{l}\right) R_{j k}$

$$
\begin{aligned}
& =\left[\nabla_{i} \nabla_{j} R_{i k}-\left(\nabla_{i} R_{i l k j}\right) f_{l}-R_{i l k j} f_{l i}\right] R_{j k} \\
\frac{1}{2} \Delta|R c|^{2} & =\frac{1}{2} \Delta\left(R_{j k} R_{j k}\right)=\nabla_{i}\left(\nabla_{i} R_{j k} R_{j k}\right) .
\end{aligned}
$$

$$
\frac{1}{2} \Delta|R c|^{2}=\left(\Delta R_{j k} R_{j k}\right)+|\nabla R c|^{2} .
$$

Further we have $\frac{1}{2} \int \Delta|R c|^{2} e^{-f}=\frac{1}{2} \int|R c|^{2} \Delta e^{-f}$. So,

$$
\begin{aligned}
\frac{1}{2} \int|R c|^{2} \Delta e^{-f} & =\int<\Delta R c, R c>e^{-f}+\int|\nabla R c|^{2} e^{-f} . \\
\frac{1}{2} \int|R c|^{2} \Delta e^{-f} & =\int|\nabla R c|^{2} e^{-f}+\int\left(\nabla_{i} \nabla_{j} R_{i k} R_{j k}-\nabla_{j} \nabla_{i} R_{i k} R_{j k}\right) e^{-f} \\
& +\int \nabla_{j} \nabla_{i} R_{i k} R_{j k} e^{-f}-\int \nabla_{i} R_{i l k j} f_{l} R_{j k} e^{-f}-\int R_{i l k j} f_{l i} R_{j k} e^{-f} \\
& =\int|\nabla R c|^{2} e^{-f}+\int K e^{-f}+\int \nabla_{j} \nabla_{i} R_{i k} R_{j k} e^{-f} \\
& -\int \nabla_{i} R_{i l k j} f_{l} R_{j k} e^{-f}-\int R_{i l k j} f_{l i} R_{j k} e^{-f} .
\end{aligned}
$$

Where $K=\left(\nabla_{i} \nabla_{j} R_{i k}-\nabla_{j} \nabla_{i} R_{i k}\right) R_{j k}$. Using Lemma 2.4 we have

$$
\begin{aligned}
\frac{1}{2} \int \Delta|R c|^{2} e^{-f} & =\int|\nabla R c|^{2} e^{-f}+\int K e^{-f}-\int \nabla_{i} R_{i l k j} f_{l} R_{j k} e^{-f} \\
& -\int R_{i l k j} f_{l i} R_{j k} e^{-f}
\end{aligned}
$$

For conformal Ricci soliton $f_{l i}=\left(\frac{1}{2 \tau}-\frac{2}{n}-p\right) g_{l i}-R_{l i}$. Putting this in (3.5) we get $\frac{1}{2} \int|R c|^{2} \Delta e^{-f}=\int|\nabla R c|^{2} e^{-f^{n}}+\int K e^{-f}-\int \nabla_{i} R_{i l k j} f_{l} R_{j k} e^{-f}$ $-\int R_{i l k j}\left[\left(\frac{1}{2 \tau}-\frac{2}{n}-p\right) g_{l i}-R_{l i}\right] R_{j k} e^{-f}$ or

$$
\begin{aligned}
\frac{1}{2} \int g^{l i}|R c|^{2} \Delta e^{-f} & =\int g^{l i}|\nabla R c|^{2} e^{-f}+\int K g^{l i} e^{-f}-\int g^{l i} \nabla_{i} R_{i l k j} f_{l} R_{j k} e^{-f} \\
& -\int g^{l i} R_{i l k j}\left(\frac{1}{2 \tau}-\frac{2}{n}-p\right) g_{l i} R_{j k} e^{-f}+\int g^{l i} R_{i l k j} R_{l i} R_{j k} e^{-f}
\end{aligned}
$$




$$
\begin{aligned}
\frac{1}{2} \int g^{l i}|R c|^{2} \Delta e^{-f} & =\int g^{l i}|\nabla R c|^{2} e^{-f}+\int K g^{l i} e^{-f}-\int g^{l i} \nabla_{i} R_{i l k j} f_{l} R_{j k} e^{-f} \\
& -\left(\frac{n}{2 \tau}-1-p n\right) \int R_{i l k j} R_{j k} e^{-f} .
\end{aligned}
$$

Acknowledgements. We would like to thank honorable referee for valuable suggestions to improve the paper.

\section{REFERENCES}

1. CAO, X. - Compact gradient shrinking Ricci solitons with positive curvature operator, J. Geom. Anal., 17 (2007), 425-433.

2. Chow, B.; Lu, P.; Ni, L. - Hamilton's Ricci flow, Graduate Studies in Mathematics, 77, American Mathematical Society, Providence, RI; Science Press, New York, 2006.

3. Fischer, A.E. - An introduction to conformal Ricci flow, http://arxiv.org/abs/math/0312519, 2003, 1-52.

4. Hamilton, R.S. - Three-manifolds with positive Ricci curvature, J. Differential Geom., 17 (1982), 255-306.

5. Müller, R. - Differential Harnack inequalities and the Ricci flow, EMS Series of Lectures in Mathematics, European Mathematical Society (EMS), Zürich, 2006.

6. Perelman, G. - The entropy formula for the Ricci flow and its geometric applications, htt://arXiv.org/abs/math/0211159, 2002, 1-39.

7. Perelman, G. - Ricci flow with surgery on three manifolds, http://arxiv.org/abs/math/0303109 2003, 1-22.

Received: 29.III.2012

Revised: 7.IX.2012

Revised: 24.I.2013

Accepted: 18.1 V.2013
Department of Mathematics, Jadavpur University, Kolkata-700032, INDIA

bhattachar1968@yahoo.co.in nirabhra.basu@gmail.com 